Chirurg 2014 · 85:639

DOI 10.1007/s00104-014-2792-0

Online publiziert: 19. Juni 2014

(c) Springer-Verlag Berlin Heidelberg 2014

H. Dralle $\cdot$ I. Satiroglu

Universitätsklinik für Allgemein-, Viszeral- und Gefäßchirurgie, Medizinische Fakultät,

Universitätsklinikum Halle (Saale)

\title{
Endoskopische Submukosaresektion kleiner neuroendokriner Tumoren des Rektums
}

\section{Originalpublikation}

Zhou X, Xie H, Xie L et al (2014) Endoscopic resection therapies for rectal neuroendocrine tumors: a systematic review and meta-analysis. J Gastroenterol Hepatol 29:259-268

\section{Hintergrund und Fragestellung}

Die Inzidenz rektaler neuroendokriner Tumoren (NET) hat in den letzten Jahren infolge verbesserter und vermehrt eingesetzter endoskopischer Untersuchungen erheblich zugenommen. Entscheidendes Kriterium für ein konventionell operatives bzw. transanal endoskopisches Vorgehen ist die Tumorgröße $<2 \mathrm{~cm}$ und ein bildgebend (Computertomographie [CT], endoskopischer Ultraschall [EUS]) negativer Metastasenbefund. Ausgangspunkt für die vorliegende Metaanalyse war die Frage, welche der gegenwärtig zur Verfügung stehenden endoskopischen Techniken mit der niedrigsten Lokalrezidivrate verbunden war.

\section{Methoden}

In die Metaanalyse wurden insgesamt 519 englischsprachige bis Februar 2013 publizierte Studien einbezogen, die mit entsprechenden Suchworten (rektale neuroendokrine Tumoren, rektale Karzinoide) in Pubmed, Embase, Medline und Cochrane Central Libary gelistet waren. Am Ende waren 10 Studien geeignet, die einen kompletten Datensatz einschließlich Nachbeobachtung und einen Vergleich von mindestens zwei der drei untersuchten endoskopischen Verfahren ermöglichen:

- konventionelle endoskopische

Mukosaresektion (EMR),
- EMR mit zusätzlichen Hilfsmitteln zur Bildung eines Pseudopedikels (Bandligatur, Saugkappe; mEMR) und

- endoskopische Submukosadissektion (ESD).

Studien, bei denen Lymphknotenmetastasen pathohistologisch nachgewiesen oder bildgebend durch CT oder EUS nicht auszuschließen waren oder eine Muskularisinvasion vorlag, wurden ausgeschlossen.

\section{Ergebnisse}

Die 10 verwertbaren Studien umfassten 650 Patienten mit rektalen NET. Bei allen Studien handelte es sich um retrospektive Studien, alle Studien waren asiatischer Provenienz (Japan, Korea, China). Die Tumorgröße betrug bis zu $15 \mathrm{~mm}$ unabhängig vom endoskopischen Verfahren. Zusammengefasst ergab der Vergleich der drei endoskopischen Verfahren folgende Ergebnisse:

- Die R0-Resektionsrate war am besten in der ESD-Gruppe, am schlechtesten in der EMR-Gruppe, die Ergebnisse der ESD- und mEMR-Gruppe waren vergleichbar.

- Die Verfahrensdauer war in der ESDGruppe am längsten, in der EMRGruppe am kürzesten, ESD und mEMR waren wiederum vergleichbar.

- Alle drei endoskopischen Verfahren hatten eine niedrige Nachblutungsund Perforationsrate $(0,6-1,4 \%)$. Die Nachblutungen und Perforationen konnten in allen Fällen endoskopisch versorgt werden.

- Lokalrezidive traten in 1,5\% der Fälle nach EMR, nicht jedoch nach den anderen beiden Endoskopieverfahren auf.

\section{Diskussion und Fazit}

Endoskopische Verfahren zur Behandlung rektaler NET sind nicht neu, es fehlen jedoch bislang in den Leitlinien der Europäischen Gesellschaft für Neuroendokrine Tumoren (ENETS) und der gleichnamigen Nordamerikanischen Gesellschaft (NANETS) Empfehlungen hinsichtlich des am besten geeigneten endoskopischen Verfahrens. Die hier vorgestellte Metaanalyse ist die bislang umfassendste bezüglich der drei analysierten Verfahren (EMR; mEMR; ESD). Studien mit Einsatz der transanalen endoskopischen Mikrochirurgie (TEM) wurden nicht einbezogen, lassen aber aufgrund aktueller Untersuchungen den Schluss zu, dass TEM, ESD und mEMR vergleichbar sind. Als Fazit der Metaanalyse kann geschlussfolgert werden, dass die endoskopische Submukosadissektion und die mEMR kleiner ( $<15 \mathrm{~mm}$ ) rektaler NET der EMR onkologisch überlegen sind. Die Indikation ist beschränkt auf Tumoren ohne Muskularisinvasion oder Lymphknotenbefall. Da die Häufigkeit des Lymphknotenbefalls mit der Tumorgröße korreliert, müsste durch weitere Studien geklärt werden, wo die obere Primärtumorgröße für ein endoskopisches Vorgehen liegt.

\section{Korrespondenzadresse}

Prof. Dr. Dr. h.c. H. Dralle

Universitätsklinik für Allgemein-, Viszeralund Gefäßchirurgie, Medizinische Fakultät, Universitätsklinikum Halle (Saale), Ernst-Grube-Str. 40, 06097 Halle (Saale) henning.dralle@uk-halle.de

Interessenkonflikt. H. Dralle und I. Satiroglu geben an, dass kein Interessenkonflikt besteht. 